\title{
RELAÇÃO DE VAZÃO DE AR E ALTURA DE LEITO COMO CRITÉRIO DE ESCALONAMENTO NO CULTIVO EM ESTADO SÓLIDO DE ASPERGILLUS NIGER EM BIORREATOR DE COLUNA
}

\author{
R. G. BASTOS ${ }^{1}$, M. P.C. VOLPI ${ }^{1}$ e D. V. MORAIS ${ }^{1}$ \\ ${ }^{1}$ Universidade Federal de São Carlos, Centro de Ciências Agrárias \\ E-mail para contato: reinaldo@cca.ufscar.br
}

\begin{abstract}
RESUMO - Apesar do grande potencial dos cultivos em estado sólido (CES) para obtenção de produtos biotecnológicos e aproveitamento de resíduos agroindustriais, não existem critérios de escalonamento específicos para estes processos. Neste contexto, a presente pesquisa teve por objetivo avaliar a razão de aeração em coluna (relação entre a vazão de ar de alimentação por altura de leito de sólidos) como critério de escalonamento para o CES de Aspergillus niger em bagaço de cana-de-açúcar. Os resultados indicaram que a manutenção de $0,00667 \mathrm{~m}^{3}$ de ar por metro de leito por minuto (vazão de ar de $3 \mathrm{~L}$ $\mathrm{min}^{-1}$ ) levou a uma produtividade máxima em ácido cítrico de $156 \mathrm{mg} \mathrm{L}^{-1} \mathrm{dia}^{-1}$, metabólito que depende da disponibilidade de oxigênio. Nesse sentido, verificou-se que a máxima taxa de consumo de oxigênio em torno de $0,044 \mathrm{mmol} \mathrm{h}^{-1}$ foi obtida nessas condições para os primeiros dias de cultivo. Os resultados indicam a importância da seleção de vazão de ar e altura de leito para o CES em biorreatores de coluna.
\end{abstract}

\section{INTRODUÇÃO}

Cultivo em estado sólido (CES) pode ser definido como crescimento de micro-organismos em uma matriz sólida não solúvel impregnada ou não com solução nutriente, em condições próximas a ausência de água livre (Pandey et al, 2000; Pandey, 2003; Couto e Sanromán, 2006). Este processo tem apresentado grande potencial para obtenção de produtos biotecnológicos, assim como para o aproveitamento de resíduos agroindustriais. Apesar disto, não existem critérios de escalonamento específicos para estes processos. $\mathrm{O}$ entendimento das questões relacionadas com o scale-up dos CES tem aumentado consideravelmente nos últimos anos e várias estratégias têm sido propostas, embora geralmente qualitativa e sem comprovação na prática (Mitchell et al, 2000). Além disso, os modelos matemáticos que descrevem os fenômenos de transferência de massa e calor em biorreatores são bastante úteis, mais não existe a proposta de critério prático que permita a avaliação. Sendo assim, considerando a importância da transferência de oxigênio em biorreatores de coluna para CES, a presente pesquisa propõe a análise do aumento de escala considerando a manutenção da relação entre a vazão de ar de alimentação e altura do leito de sólidos, geralmente limitante. 
A utilização de bagaço de cana-de-açúcar como suporte para os CES vem sendo citada na literatura, desde que as partículas sejam impregnadas e umidificadas com uma solução nutriente adequada (Kumar et al., 2003). De acordo com Pandey e Soccol (1998), o bagaço de cana consiste de água (46-52\% em massa), fibras (43 - 52\%), incluindo celulose (50\%), hemicelulose $(25 \%)$ e lignina $(25 \%)$; e pequena quantidade de sólidos solúveis (2- 6\%). Apesar do uso do bagaço de cana para gerar energia nas usinas de açúcar e álcool no Brasil, é produzida considerável quantidade em excesso, o que poderia levar a um grave problema ambiental. A vinhaça representa a sua principal água residuária, sendo gerada pelos atuais métodos de fermentação e destilação da ordem de $10 \mathrm{~L}$ para cada litro de etanol produzido. O aproveitamento deste líquido nutriente para impregnar o bagaço de cana-de-açúcar representa o desenvolvimento de um processo inovador e o aproveitamento combinado de dois subprodutos gerados na mesma plataforma industrial. Além disso, não existem relatos da utilização de águas residuárias para impregnar matrizes sólidas visando o CES.

Os fungos são geralmente os micro-organismos mais utilizados nos CES devido às características físicas e bioquímicas do processo, o qual é conduzido em baixas condições de atividade de água. Nesse sentido, Aspergillus niger é um dos micro-organismos mais amplamente estudado e utilizado em processos industriais, com destaque para produção de ácido cítrico e enzimas. Devido às suas características fisiológicas e amplo conhecimento dos seus parâmetros cinéticos de crescimento, é importante a sua aplicação no desenvolvimento e estudo de CES não convencionais.

Ácido cítrico é um importante produto comercial com produção global em torno de 1 milhão de toneladas, sendo que a maior parte é produzida por cultivos microbianos em meios líquidos contendo amido ou sacarose (Vandenberghe et al, 2000; Khosravi-Darani e Zoghi, 2008). A indústria de alimentos é a principal consumidora de ácido cítrico (cerca de 70\%), seguido do setor farmacêutico com $12 \%$. Em certos micro-organismos, como Aspergillus niger, sob determinadas condições, o acúmulo de ácido cítrico se dá devido a uma falha no Ciclo de Krebs, além da presença de íons metálicos como cofatores enzimáticos (Angumeenal e Venkappayya, 2013).

Considerando a viabilidade da produção de ácido cítrico por Aspergillus niger relatado na literatura (Kumar et al., 2003; Kumar e Jain, 2008) e em estudos anteriores do nosso grupo de pesquisa (Bastos, 2012; Franco e Bastos, 2012; Oliveira et al., 2012), o objetivo deste trabalho foi estender estes estudos propondo um critério de escalonamento relacionando a vazão de ar com a altura do leio para a produção de ácido cítrico em biorreator de leito fixo a partir de bagaço de cana-de-açúcar impregnado com vinhaça. Para isso, partiu-se de condições previamente selecionadas em trabalhos anteriores e foi utilizado um biorreator de coluna de leito fixo encamisado para controle da temperatura, com volume de meio dez vezes superior e pontos axiais para amostragem de sólidos e gases. Os resultados foram analisados em termos de produção e rendimento de glicose em ácido cítrico, além da demanda de oxigênio na posição axial do biorreator. 


\section{MATERIAL E MÉTODOS}

\subsection{Inóculo}

O inóculo de Aspergillus niger foi mantido em meio PDA a $4^{\circ} \mathrm{C}$, sendo propagados por sete dias em frascos Erlenmeyer's com meio PDA anterior aos experimentos em biorreator de coluna.

\subsection{Suporte sólido}

A vinhaça e o bagaço de cana-de-açúcar foram coletados de uma indústria sucroalcooleira da região de Araras, sendo levados ao Laboratório de Microbiologia Aplicada (LABMAC/CCA/UFSCar). O bagaço foi sendo classificado em conjunto de peneiras Tyler, sendo utilizadas as partículas de diâmetro entre 1,17 e $0,59 \mathrm{~mm}$. Tanto a vinhaça como o bagaço selecionado serão esterilizados em autoclave a $121^{\circ} \mathrm{C}$ por 20 minutos.

\subsection{Ensaios}

Os experimentos foram conduzidos em biorreator de leito fixo de $50 \mathrm{~cm}$ de altura com 5 $\mathrm{cm}$ de diâmetro, controle de temperatura por camisa térmica e pontos axiais de coleta de amostra de sólidos e análise do gás a cada $10 \mathrm{~cm}$ de altura de leito.

Os ensaios partiram de condições selecionadas em experimentos anteriores desenvolvidos pelo nosso grupo de pesquisa em Colunas de Raimbault com volume de meio sólido dez vezes inferior (Bastos, 2012; Franco e Bastos, 2012; Oliveira et al., 2012), ou seja, $25^{\circ} \mathrm{C}$, vazão de ar selecionada de $0,8 \mathrm{~L} \mathrm{~min}^{-1}$. Além disso, foi avaliado o desempenho do CES no valor calculado para manter a razão de aeração, ou seja, a relação do volume de ar pela altura de leito de leito de sólidos por $\min \left(3 \mathrm{~L} \mathrm{~min}^{-1}\right)$.

\subsection{Análises}

O extrato fúngico foi obtido com água e acetona $50 \%$ (45 minutos por estágio) e $28^{\circ} \mathrm{C}$ em agitador orbital a $150 \mathrm{rpm}$, em condições adaptadas de Khoshavi-Darani e Zhogi (2008)e otimizadas por nosso grupo de pesquisa. O extrato fúngico bruto obtido foi então caracterizado quanto ao $\mathrm{pH}$ por potenciometria, glicose pelo método enzimático glicose oxidase - peroxidase, com kit da LABORLAB ${ }^{\circledR}$ e teor de ácido cítrico por reação colorimétrica com piridina e anidrido acético usando kit comercial da In Vitro ${ }^{\circledR}$. A partir destes dados, foram calculadas a produtividade em termos de ácido cítrico e o rendimento a partir de glicose.

A composição do ar de entrada e saída das colunas em termos de oxigênio foi medida por oxímetro modelo YSI ${ }^{\circledR} 55-\mathrm{D}$ acoplado ao sistema. 


\section{RESULTADOS E DISCUSSÃO}

A Figura 1 apresenta os perfis axiais de ácido cítrico produzido para ensaios em coluna com aeração de $0,8 \mathrm{~L} \mathrm{~min}^{-1}$. Os resultados indicam um comportamento similar de produção de ácido cítrico até três dias nas duas posições axiais, sendo que a partir deste momento a curva a $100 \mathrm{~mm}$ tende a uma valor máximo, enquanto para $200 \mathrm{~mm}$ ocorre uma desaceleração, sugerindo condições limitadas de oxigênio.

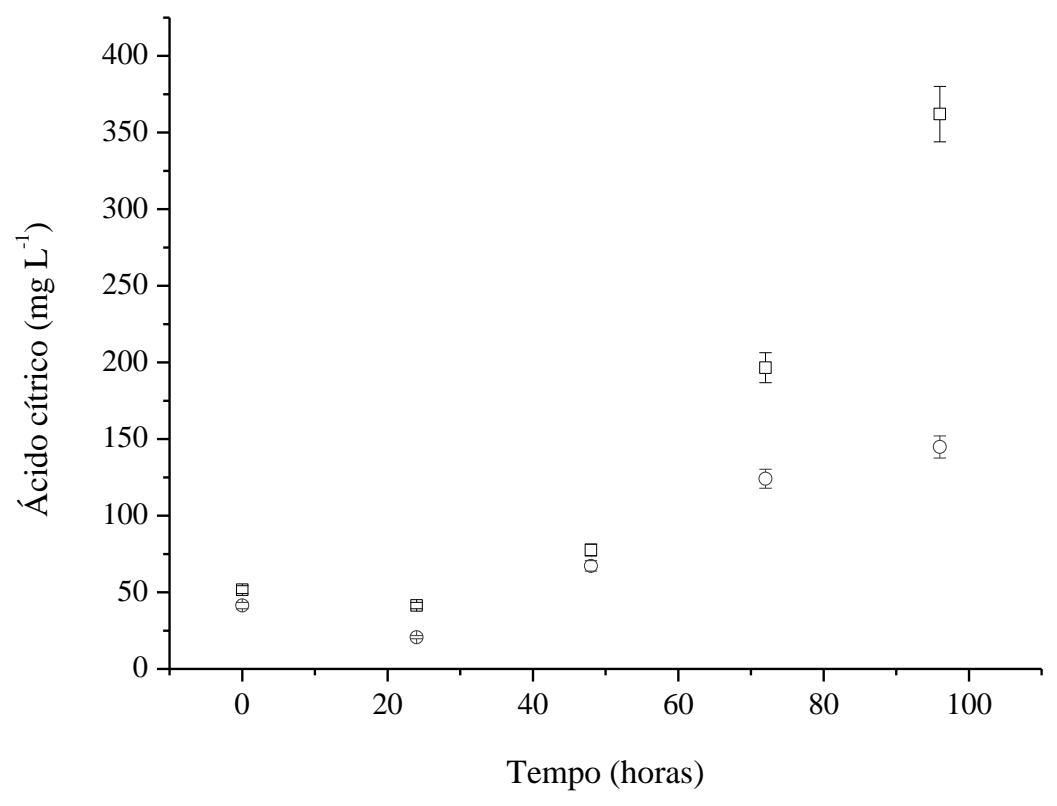

Figura 1 - Perfis axiais de ácido cítrico no extrato fúngico obtidos para o CES de Aspergillus niger em bagaço de cana-de-açúcar com vinhaças para vazões de aeração de $0,8 \mathrm{~L} \mathrm{~min}^{-1} \mathrm{em} 100$ (口) e $200 \mathrm{~mm}(\mathrm{O})$ de altura de leito

Tal limitação leva a produtividade de ácido cítrico de 3,77 mg (L.h $)^{-1}$ a $100 \mathrm{~mm}$ e $1,51 \mathrm{mg}$ (L.h) $)^{-1}$ a $200 \mathrm{~mm}$. Além disso, os rendimentos de glicose em ácido cítrico foram $0,73 \mathrm{~g} \mathrm{~g}^{-1} \mathrm{a}$ $100 \mathrm{~mm}$ e $0,33 \mathrm{~g} \mathrm{~g}^{-1}$ a $200 \mathrm{~mm}$. A concentração máxima de ácido cítrico obtida encontra-se próxima dos valores encontrado, apensar da produtividade inferior (Khoshavi-Darani e Zhogi, 2008). Entretanto, cabe ressaltar que os autores citados realizaram experimentos a partir impregnado as partículas com meio nutriente e em condições otimizadas de pré-tratamento do de bagaço de cana-de-açúcar. Sendo assim, por tratar-se de uma etapa onde já houve aumento de produção de um CES com bagaço combinado com vinhaça, os resultados são promissores quanto comparados com os dados reportados na literatura. Uma produção maior seria atingida com uma disponibilidade maior de glicose na solução nutriente. Como a vinhaça usada para impregnar o 
meio sólido não apresenta altos teores de glicose (Bastos et al., 2009), o fungo precisa hidrolisar parte do bagaço, o que torna o metabolismo mais lento e leva a produtividades menores.

Por outro lado, se analisarmos os resultados para a vazão de $3 \mathrm{~L} \mathrm{~min}^{-1}$ (Figura 2), a qual fisicamente mantém as mesmas condições de aeração das colunas menores, tem-se a produtividade máxima de ácido cítrico de 2,29 mg (L.h) ${ }^{-1}$ a $100 \mathrm{~mm}$ e 8,46 mg (L.h) ${ }^{-1}$ a $200 \mathrm{~mm}$, com os rendimentos a partir de glicose de $0,16 \mathrm{~g} \mathrm{~g}^{-1}$ a $100 \mathrm{~mm}$ e 2,26 $\mathrm{g} \mathrm{g}^{-1}$ a $200 \mathrm{~mm}$. Este valor de rendimento superior a 1 indica que não apenas a glicose proveniente da vinhaça está sendo consumida para produção de biomassa e metabólitos. O fungo certamente está hidrolisando parte dos polissacarídeos estruturais do suporte sólido e este substrato não está sendo ponderado no cálculo do rendimento. Esta avaliação pode ser feita considerando que a análise determinação de glicose é enzimática, isto é, análise que quantifica apenas este monossacarídeo e não outros açúcares redutores. Este fenômeno ocorre principalmente nas primeiras horas de cultivo em aerobiose, haja vista as curvas de demanda de oxigênio apresentadas na Figura 3. A diferença entre os valores para 100 e $200 \mathrm{~mm}$ de altura de leito nas primeiras horas reflete o maior volume de meio sólido e, consequentemente, uma quantidade de micro-organismos e demanda de oxigênio duas vezes superior. Após este período, há uma tendência de equilíbrio até mesmo devido a dificuldade maior de transferência de oxigênio ao longo do leio de sólidos.

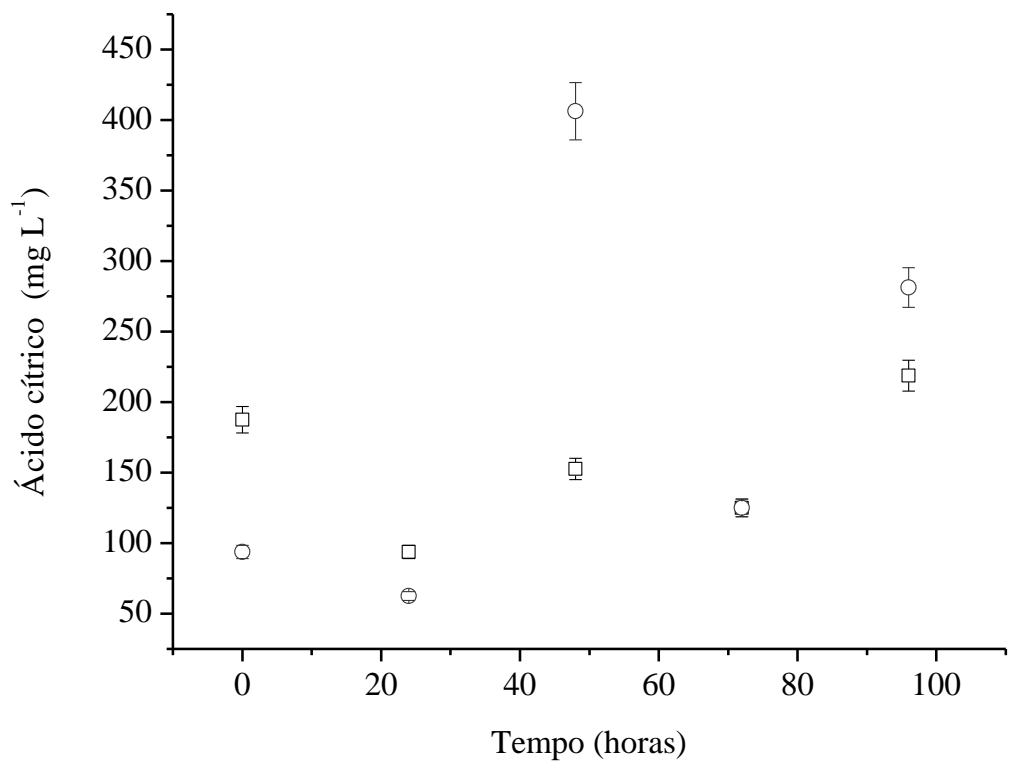

Figura 2 - Perfis axiais de ácido cítrico no extrato fúngico obtidos para o CES de Aspergillus niger em bagaço de cana-de-açúcar com vinhaças para vazões de aeração de $3 \mathrm{~L} \mathrm{~min}^{-1}$ em 100 (口) e $200 \mathrm{~mm}$ (O) de altura de leito 


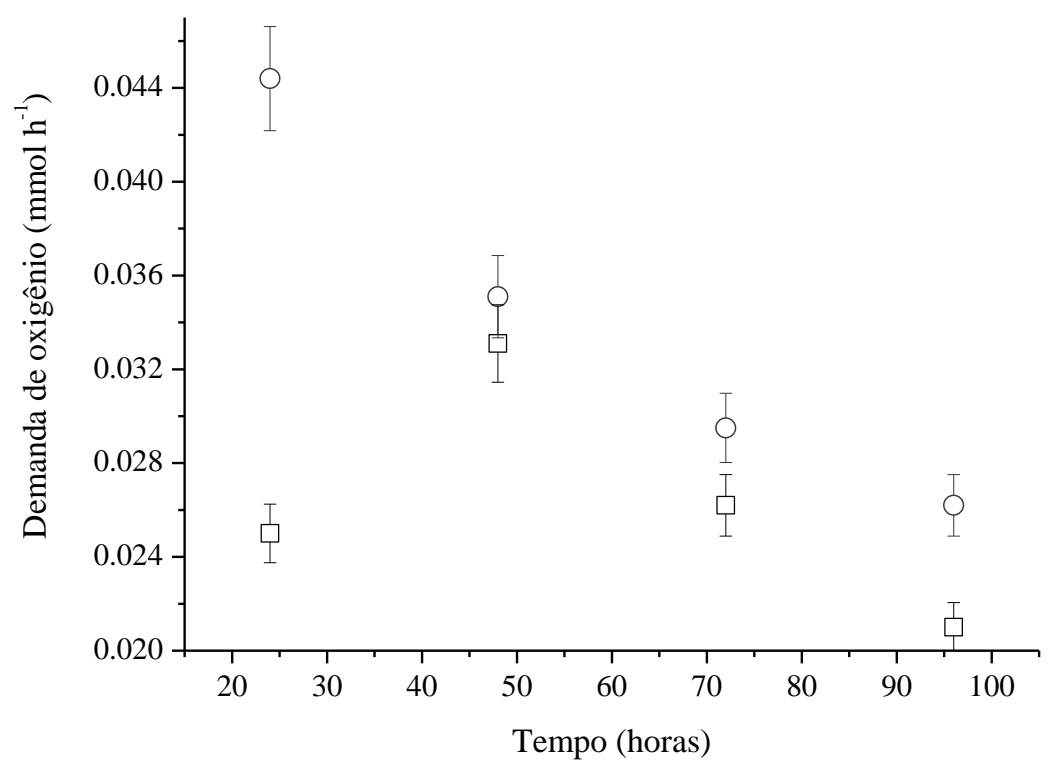

Figura 3 - Demanda de oxigênio no CES de Aspergillus niger em bagaço de cana-de-açúcar com vinhaças para $3 \mathrm{~L} \mathrm{~min}^{-1}$ de vazão de ar nas posições axiais $100(\square)$ e $200 \mathrm{~mm}(\mathrm{O})$ de altura de leito

Considerando os dados do trabalho de Ikasari e Mitchell (1998), o CES de Aspergillus niger em bagaço de cana-de-açúcar com vinhaça apresenta uma taxa específica de consumo de oxigênio estimada de $0,008 \mathrm{mg} \mathrm{O}_{2}$ por $\mathrm{mg}$ de biomassa por hora, o que sugere uma baixa demanda e condições de não limitação de oxigênio.

Vandenberghe et al (2000) reportaram produções de $88 \mathrm{~g}$ de ácido cítrico por $\mathrm{kg}$ de massa seca para bagaço de mandioca, $48,7 \mathrm{~g} \mathrm{~kg}^{-1}$ para bagaço de cana-de-açúcar e $12,7 \mathrm{~g} \mathrm{~kg}^{-1}$ para casca de café. No ponto de concentração máxima de ácido cítrico a $200 \mathrm{~mm}$ de altura de leito com aeração de $3 \mathrm{~L} \mathrm{~min}^{-1}$, foi obtida uma produção de $27,1 \mathrm{~g} \mathrm{~kg}^{-1}$ bagaço de cana-de-açúcar, valor ainda inferior às informações da literatura, mas promissor uma vez que é usada apenas vinhaça para impregnar o suporte sólido.

Sendo assim, considerando a produção em termos de ácido cítrico obtida a partir de dois subprodutos do setor sucroenergético aliada a demanda de oxigênio exigida, normalmente uma limitação nos CES, os resultados indicam sucesso no escalonamento deste cultivo a partir do critério de aeração e altura para colunas de leito fixo. 


\section{CONCLUSÕES}

Nas condições experimentais foi possível concluir que é viável a produção de ácido cítrico por Aspergillus niger em bagaço de cana-de-açúcar com vinhaça, com baixa demanda de oxigênio, sendo que a relação entre vazão de ar e altura de leito pode ser usada como critério de escalonamento deste processo.

\section{REFERÊNCIAS}

ANGUMEENAL, A.R.; VENKAPPAYYA, D. An overview of citric acid production. LWT Food Science and Technology. V. 50, p. 357-370. 2013.

BASTOS, R.G. Solid state cultivation of Aspergillus niger for citric acid and AMG production on sugarcane bagasse with vinasse. Proceedings of $9^{\text {th }}$ World Congress on Industrial Biotechnology and Bioprocess. BIO - Biotechnology Industry Organization. Orlando, Estados Unidos. 2012.

BASTOS, R.G.; RIZZO, P.; PARAZZI, C.; VALSECHI, O.A. Cultivo mixotrófico e heterotrófico de cianobactéria em água residuária da indústria setor sucroenergética. XVII Simpósio Nacional de Bioprocessos (SINAFERM), Natal/RN, Brazil. 2009.

COUTO, S. R.; SANROMÁN, M. Á. Application of solid-state fermentation to food industry-A review. Journal of Food Engineering, 76(3), 291-302, 2006.

FRANCO, F.M.; BASTOS, R.G. Efeito da umidade inicial na produção de ácido cítrico por Aspergillus niger a partir de bagaço de cana-de-açúcar e vinhaça. XIX Congresso Brasileiro de Engenharia Química (COBEQ), Búzios/RJ. 2012.

IKASARI, L.; MITCHELL, D. A. Oxygen uptake kinetics during solid state fermentation with Rhizopus oligosporus. Biotechnology Techniques, 12(2), 171-175, 1998.

KHOSRAVI-DARANI, K.; ZOGHI, A. Comparison of pretreatment strategies of sugarcane bagasse: experimental design for citric acid production. Bioresource Technology, V. 99, p. 6986-6993, 2008.

KUMAR, A.; JAIN, V.K. Solid state fermentation studies of citric acid production. African Journal of Biotechnology, V.7, p. 644-650, 2008.

KUMAR, D.; JAIN, V.K.; SHANKER, G.; SRIVASTAVA, A. Citric acid production by solid state fermentation using sugarcane bagasse. Process Biochemistry 38, 1731-1738, 2003.

MITCHELL, D. A.; KRIEGER, N.; STUART, D. M.; PANDEY, A. New developments in solidstate fermentation II . Rational approaches to the design , operation and scale-up of bioreactors, Process Biochemistry 35, 1211-1225, 2000. 
OLIVEIRA, A.F.; MATOS, V.C.; BASTOS, R.G. Cultivation of Aspergillus niger on sugarcane bagasse with vinasse. Bioscience Journal, v. 28, n. 6, p. 889-894. 2012

PANDEY, A. Solid state fermentation. Biochemical Engineering Journal. V.13.p.81-84. 2003.

PANDEY, A.; SOCCOL, C.R. Bioconversion of biomass: a case of lignocellulosics bioconversions in solid state fermentation. Braz. Arch Biol. Technol. 41, 379-390, 1998.

PANDEY, A.; SOCCOL, C. R.; MITCHELL, D. New developments in solid state fermentation: I-bioprocesses and products. Process Biochemistry 35(10), 1153-1169, 2000.

VANDENBERGHE, L. P.; SOCCOL, C. R.; PANDEY, A.; LEBEAULT, J.-M. Solid-state fermentation for the synthesis of citric acid by Aspergillus niger. Bioresource Technology, 74(2), 175-178, 2000. 